\title{
Short-term training alters the control of mitochondrial respiration rate before maximal oxidative ATP synthesis
}

\author{
Gwenael Layec $^{1,2}$, Luke J. Haseler ${ }^{3}$, Jan Hoff ${ }^{4}$, Corey R. Hart ${ }^{1,2,5}$, Xin Liu' ${ }^{6}$, Yann Le Fur ${ }^{7}$, \\ Eun-Kee Jeong $^{6}$, and Russell S. Richardson ${ }^{1,2,5}$ \\ ${ }_{1}^{1}$ Department of Medicine, Division of Geriatrics, University of Utah, Salt Lake City, Utah, USA \\ ${ }^{2}$ Geriatric Research, Education, and Clinical Center, George E. Whalen VA Medical Center, Salt \\ Lake City, Utah, USA \\ ${ }^{3}$ Heart Foundation Research Centre, Griffith Health Institute, Griffith University, Queensland \\ Australia \\ ${ }^{4}$ Department of Medicine, Norwegian University of Science and Technology, Trondheim, Norway \\ ${ }^{5}$ Exercise and Sport Science, University of Utah, Salt Lake City, Utah, USA \\ ${ }^{6}$ Department of Radiology and Utah Center for Advanced Imaging Research, University of Utah, \\ Salt Lake City, Utah, USA \\ ${ }^{7}$ Centre de Resonance Magnetique Biologique et Medicale, UMR CNRS 7339, Faculté de \\ Médecine de Marseille, Marseille, France
}

\section{Abstract}

\begin{abstract}
Aim-Short-term exercise training may induce metabolic and performance adaptations before any changes in mitochondrial enzyme potential. However, there has not been a study that has directly assessed changes in mitochondrial oxidative capacity or metabolic control as a consequence of such training in vivo. Therefore, we used ${ }^{31} \mathrm{P}$-magnetic resonance spectroscopy $\left({ }^{31} \mathrm{P}-\mathrm{MRS}\right)$ to examine the effect of short-term plantar flexion exercise training on phosphocreatine ( $\mathrm{PCr}$ ) recovery kinetics and the control of respiration rate.
\end{abstract}

Method-To this aim, we investigated 12 healthy men, experienced with this exercise modality (TRA), and 7 time-control subjects (TC).

Results-After 5 days of training, maximum work rate during incremental plantar flexion exercise was significantly improved $(P<0.01)$. During the recovery period, the maximal rate of oxidative ATP synthesis (PRE: $28 \pm 13 \mathrm{mM} \cdot \mathrm{min}^{-1}$; POST: $26 \pm 15 \mathrm{mM} \cdot \mathrm{min}^{-1}$ ) and the PCr recovery time constant (PRE: $31 \pm 19$ s; POST: $29 \pm 16$ ) were not significantly altered. In contrast, the Hill coefficient $\left(n_{H}\right)$ describing the cooperativity between respiration rate and ADP was significantly increased in TRA (PRE: $n_{H}=2.7 \pm 1.4$; POST: $n_{H}=3.4 \pm 1.9, P<0.05$ ). Meanwhile, there were no systematic variations in any of these variables in TC.

Conclusion-This study reveals that 5 days of training induces rapid adaptation in the allosteric control of respiration rate by ADP before any substantial improvement in muscle oxidative capacity occurs.

Corresponding author: G. Layec, VA Medical Center, Bldg 2, 500 Foothill Dr., Salt Lake City, Utah 84148, USA, gwenael.layec@utah.edu.

Disclosures:

No conflicts of interest, financial or otherwise, are declared by the author(s). 


\section{Keywords}

skeletal muscle energetics; mitochondrial function; exercise training; metabolic control; 31P-MRS

\section{Introduction}

Regular endurance exercise training induces a myriad of muscle metabolic and systemic adaptations (Holloszy \& Coyle, 1984; Delp, 1998; Jones \& Carter, 2000). For example, it is traditionally thought that the increase in mitochondrial respiration capacity associated with exercise training leads to smaller relative changes in the cytosolic modulators of oxidative phosphorylation (NADH, ADP, Pi and PCr), implying an increased respiration sensitivity of the mitochondria (Dudley et al., 1987). Indeed, it is reasoned that muscle tissue with higher mitochondrial density requires less $\mathrm{O}_{2}$ uptake and ATP synthesis per mitochondrion for a given energy demand. Thus, at a given work rate, trained muscle would require a lower amount of ADP and Pi as well as reduced $\mathrm{PCr}$ depletion than untrained muscle. Of interest, changes in muscle energetics appear to occur even after a short period of endurance training (5-10 days), with a reduction in exercise-induced PCr depletion and ADP and Pi accumulation documented following such short-term exercise training (Green et al., 1991; Putman et al., 1998).

Based on the assessment of mitochondrial enzyme activity as a marker of oxidative capacity, these aforementioned acute adaptations seem to take place in the absence of altered oxidative potential (Green et al., 1991; Putman et al., 1998). Thus, this would suggest that in the early stage of exercise training the sensitivity of mitochondrial respiration is increased before the capacity of mitochondria to respire (or mitochondrial density) has changed. It should, however, be noted that conflicting results, more in line with the traditional concept of a required increase in mitochondrial respiration capacity, have been reported (Spina et al., 1996), even by work from the same group (Green et al., 1999). This may be because changes in maximal activity of some mitochondrial enzymes might not truly reflect changes in the flux of mitochondrial respiration (Starritt et al., 1999). In this context, post-exercise $\mathrm{PCr}$ recovery kinetics measured by ${ }^{31} \mathrm{P}$ magnetic resonance spectroscopy $\left({ }^{31} \mathrm{P}-\mathrm{MRS}\right)$, now widely accepted as a sensitive index of muscle oxidative capacity in vivo (Thompson et al., 1995), could non-invasively provide insight into whether short-term training-induced changes in muscle energetics are associated with improved muscle oxidative capacity and/or the sensitivity of respiration rate to the factors involved in the control of oxidative phosphorylation (e.g. ADP, Pi, NADH, $\mathrm{O}_{2}$ ). In particular, this would be accompanied by in a change in the relationship between the respiration rate and [ADP], which is commonly believed to play a central role in the regulation of mitochondrial respiration (Chance \& Williams, 1955; Jeneson et al., 2009). However, the hypothesis that short-term exercise training may affect respiratory control has yet to be tested.

Therefore, the goal of the present study was to utilize ${ }^{31} \mathrm{P}-\mathrm{MRS}$ to explore the effects of short-term exercise training on the control of respiration rate by ADP and oxidative capacity in young healthy subjects during plantar flexion exercise. We hypothesized that short-term training would increase the sensitivity of mitochondrial respiration to [ADP] before any measurable change in muscle oxidative capacity.

\section{Methods}

Subject

Following informed consent procedures, 12 healthy male subjects participated in the training study (TRA; mean $\pm \mathrm{SD}$, age $24 \pm 6$; weight, $78 \pm 8 \mathrm{~kg}$; height, $178 \pm 7 \mathrm{~cm}$ ) and 7 additional 
subjects acted as time-controls (TC; mean $\pm \mathrm{SD}$, age $22 \pm 2$; weight, $72 \pm 7 \mathrm{~kg}$; height, 180 $\pm 4 \mathrm{~cm}$ ). The study was approved by the Human Research Protection Program of the University of California, San Diego.

\section{Exercise protocol}

Subjects were already accustomed to plantar flexion exercise in the confines of a whole body MRI system through participation in prior acute studies and familiarization procedures. The maximum plantar flexion work rate (WRmax) for each subject was determined by performing a graded test to maximum effort, as previously described (Haseler et al., 1999). On a different day, subjects then performed constant-load sub-maximal plantar flexion at $\sim 50 \%$ of WRmax (frequency of $1 \mathrm{~Hz}$ ) while lying supine in the superconducting magnet (GE 1.5T Medical Systems, Milwaukee, WI). Specifically, after 2 min of rest, subjects exercised for $4 \mathrm{~min}$ at $50 \%$ of WRmax followed by $5 \mathrm{~min}$ of recovery. In order to improve the confidence of the [ADP]-respiration rate fitting and achieve a high [ADP], this protocol was followed by a supra-maximal exercise at $\sim 120 \%$ of WRmax for $1 \mathrm{~min}$ and $5 \mathrm{~min}$ of recovery. Both the graded exercise and the constant-load plantar flexion studies were repeated pre- and post-5 days of plantar flexion training. The constant-load exercise was performed at the same absolute work rate pre- and post-training.

\section{Training protocol}

Following pre-testing all subjects underwent a 5-day period of aerobic high intensity plantar flexion exercise training, utilizing an identical ergometer to that used for the NMR experiments. All training sessions started with a 5-min warm-up period consisting of low intensity plantar flexion ( $<30 \%$ of WRmax). The interval training consisted of $4 \times 4 \mathrm{~min}$ at $80 \%$ of WRmax with 3 min of active rest ( $<30 \%$ of WRmax), as previously reported (Helgerud et al., 2007). The post-testing was performed $\sim 24-48 \mathrm{~h}$ after the last training session.

31P MRS

MRS was performed using a clinical 1.5T General Electric Signa system (LX 8.3 version) operating at $25.86 \mathrm{MHz}$ for ${ }^{31} \mathrm{P} .{ }^{31} \mathrm{P}$ MRS data were acquired with a dual frequency flexible array spectroscopy coil (Medical Advances Inc., Milwaukee, Wisconsin) positioned around the calf at its maximum diameter. The phosphorus coil was an $11.5 \mathrm{~cm}$ square, centered between two $14 \times 15.5 \mathrm{~cm}$ Helmholtz-type proton coils. One fully relaxed spectrum was acquired at rest (repetition time $=30 \mathrm{~s}$ ). Then, partially relaxed spectra were acquired throughout the rest-exercise-recovery protocol with the following parameters (radiofrequency hard pulse duration $=500 \mu \mathrm{s}$, repetition time $=4 \mathrm{~s}$, number of excitation $=$ 1 , sweep width $=2.5 \mathrm{kHz}$, Data points $=1024$, nominal flip angle $=90^{\circ}$ ).

\section{Data Analysis}

Data were processed using SAGE/IDL software on a Silicon Graphics INDIGO workstation. Each free induction decay was processed with $5 \mathrm{~Hz}$ exponential line broadening prior to zero filling and Fourier transformation. All spectra were manually phased using zero and first order phase corrections. Then, relative concentrations of phosphocreatine [PCr], inorganic phosphate [Pi], phosphodiester [PDE] and [ATP] were obtained by a frequencydomain fitting routine for each peak according to a Lorentzian function using MarquardtLevenberg equation. The resting concentrations of phosphorus metabolites were calculated from the peak areas of the relaxed spectra recorded at rest and assuming an ATP concentration of $8.2 \mathrm{mM}$ at rest, as previously described (Harris et al., 1974). Muscle intracellular $\mathrm{pH}$ was calculated from the chemical shift difference () of the Pi peak relative to the PCr peak (Taylor et al., 1983). When Pi splitting was evident, the $\mathrm{pH}$ corresponding to 
each Pi pool was calculated separately as $\mathrm{pH}_{1}$ and $\mathrm{pH}_{2}$ on the basis of the chemical shift of each peak relative to $\mathrm{PCr}$. The overall muscle $\mathrm{pH}$ was then calculated as $\mathrm{pH}=\mathrm{pH}_{1}\left(\operatorname{areaPi}_{1} /\right.$ total Pi area) $+\mathrm{pH}_{2}\left(\operatorname{areaPi}_{2} /\right.$ total Pi area). The free cytosolic [ADP] was calculated from $[\mathrm{PCr}]$ and $\mathrm{pH}$ using the creatine kinase equilibrium constant $\left(K_{\mathrm{CK}}=1.66 \times 10^{9} \mathrm{M}^{1}\right)$ and assuming that phosphocreatine represents $85 \%$ of the total creatine content (Jeneson et al., 1995). $\Delta \mathrm{G}_{\text {ATP }}$ was calculated using the following equation and constants according to Kemp et al. (Kemp et al., 2001):

$$
\begin{gathered}
\Delta G_{\mathrm{ATP}}=\Delta G_{\mathrm{ATP}}^{0}+\mathrm{RT} \ln \frac{[\mathrm{ADP}] \cdot[P i]}{[\mathrm{ATP}]} \\
\Delta G_{\mathrm{ATP}}^{0}=\mathrm{RT}\left[\ln \left(K_{\mathrm{ATP}}^{\mathrm{true}}\right)+\ln \left(\frac{f_{\mathrm{ATP}} \cdot f_{\mathrm{ADP}} \cdot f_{\mathrm{Pi}}}{\left[H^{+}\right]}\right)\right]
\end{gathered}
$$

With $K_{\text {ATP }}^{\text {true }}=0.722$ (Golding et al., 1995), $\mathrm{R}=8.3145 \mathrm{~K}^{-1} \mathrm{M}^{-1}$ and $\mathrm{T}=310 \mathrm{~K}$.

The PCr recovery kinetics were determined by fitting the PCr time-dependent changes during the recovery period to a single exponential curve described by the following equation:

$$
[\mathrm{PCr}](t)=[\mathrm{PCr}]_{\mathrm{end}}+[\mathrm{PCr}]_{\mathrm{cons}}\left(1-e^{-(t / \tau p)}\right)
$$

where $[\mathrm{PCr}]_{\mathrm{end}}$ is the concentration of $[\mathrm{PCr}]$ measured at end-of-exercise and $[\mathrm{PCr}]_{\text {cons }}$ refers to the amount of $\mathrm{PCr}$ consumed at the end of the exercise session. The initial rate of $\mathrm{PCr}$ resynthesis $\left(\mathrm{Vi}_{\mathrm{PCr}}\right)$ was calculated as follows:

$$
\mathrm{Vi}_{\mathrm{PCr}}=k \cdot[\mathrm{PCr}]_{\text {cons }}
$$

in which $[\mathrm{PCr}]_{\mathrm{cons}}$, indicates the amount of $\mathrm{PCr}$ consumed at the end of exercise and the rate constant, $\mathrm{k}=1 / \tau$.

Then, using a multi-point analysis, the control of respiration rate by ADP was fitted using non-linear least square techniques from the following function (Wust et al., 2011):

$$
\mathrm{Vi}=\left(\operatorname{Vmax} \cdot[\mathrm{ADP}]^{\mathrm{nH}}\right) /\left(K_{m}^{\mathrm{nh}}+[\mathrm{ADP}]^{\mathrm{nh}}\right)
$$

In which $n_{H}$ is the Hill coefficient, $\mathrm{K}_{\mathrm{m}}$ the ADP value at half-maximal $V i$ and $\mathrm{V}_{\max }$ the maximal ADP-stimulated respiration rate (figure 4). Although, we acknowledge that even in absence of ADP, there is some mitochondrial respiration (uncoupled respiration), the minimal ADP-stimulated respiration rate $\left(\mathrm{V}_{\min }\right)$ was not included in the model as the estimation of this parameter has poor accuracy due to the low signal to noise ratio of respiration rate and [ADP] in the resting state. As a result, previous studies (Jeneson et al., 1996; Conley et al., 1998; Jeneson et al., 2009) using a 4 parameter model reported nonphysiological values for this parameter (negative values for $\mathrm{V}_{\min }$ ). In addition, reducing the numbers of parameters of the fitting (and therefore the degrees of freedom) would decrease the confidence intervals for the other estimated parameters $\left(\mathrm{V}_{\max }, \mathrm{K}_{\mathrm{m}}, n_{H}\right)$ characterizing the relationship between $\mathrm{ADP}$ and mitochondrial respiration rate.

Model variables were determined with an iterative process by minimizing the sum of squared residuals (RSS) between the fitted function and the observed values. The goodness of fit was assessed by visual inspection of the residual plot and the frequency plot 
distribution of the residuals, Chi square values, and the coefficient of determination $\left(\mathrm{r}^{2}\right)$ was calculated as follows (Motulsky \& Christopoulos, 2003):

$$
r^{2}=1-\left(\mathrm{SS}_{\mathrm{reg}} / \mathrm{SS}_{\text {tot }}\right)
$$

with SSreg, the sum of squares of the residuals from the fit and SStot, the sum of squares of the residuals from the mean.

\section{Statistical Analysis}

The difference between pre and post-tests were either examined using paired t-tests or the nonparametric Wilcoxon test, where appropriate (Statsoft, version 5.5; Statistica, Tulsa, Oklahoma). The intra-subject coefficient of variation was calculated as the standard deviation of measurements recorded during both visits divided by the mean of the two visits. Statistical significance was accepted at $P<0.05$. Results are presented as mean $\pm \mathrm{SD}$ in tables and mean \pm SEM in figures for clarity.

\section{Results}

\section{Plantar flexion exercise testing}

The power output during constant load exercise, by experimental design, remained unchanged at $6 \pm 2 \mathrm{~W}$, while plantar flexion WRmax was significantly increased after shortterm training in TRA (PRE: $13 \pm 3 \mathrm{~W}$; POST: $17 \pm 4 \mathrm{~W}, P<0.01$ ).

\section{High-energy phosphate compounds and intracellular pH}

MR spectra recorded during the rest-exercise-recovery protocol in one subject is illustrated in Figure 1. At rest, the pre-training [PCr], [Pi], [PDE] and [ADP] concentrations were $32 \pm$ $3 \mathrm{mM}, 2.9 \pm 1.3 \mathrm{mM}, 1.0 \pm 0.9 \mathrm{mM}$ and $8 \pm 2 \mu \mathrm{M}$, respectively, while resting $\mathrm{pH}$ was 7.03 \pm 0.05 . Post-training, the resting concentrations $(\mathrm{PCr}=34 \pm 4 \mathrm{mM}, \mathrm{Pi}=3.4 \pm 1.1 \mathrm{mM}$, PDE $=0.9 \pm 0.6 \mathrm{mM}$ and $\mathrm{ADP}=9 \pm 2 \mu \mathrm{M})$ as well as $\mathrm{pH}(7.05 \pm 0.05)$ were not significantly different $(P>0.05)$. Table 1 summarizes changes in intracellular metabolites and $\mathrm{pH}$ at the end of a constant-load protocol at $\sim 50 \%$ of WRmax in TRA. By the end of the second exercise bout (120\% of WRmax), [ADP] amounted to $78 \pm 62 \mu \mathrm{M}$ and $55 \pm 36 \mu \mathrm{M}$ pre- and post-training respectively, therefore covering a large range of [ADP].

\section{PCr kinetics at the offset of exercise}

Mean PCr recovery dynamics pre- and post-training are displayed in Figure 2. During the recovery period, the $\mathrm{PCr}$ offset time constant of the submaximal exercise as well as $\mathrm{V}_{\max }$ (Figure 3) were not significantly different before and after short-term training (Table 1, $P$ > $0.05)$. However, after 5 days of training, the Hill coefficient was significantly enhanced (Table 1, $P<0.05$ ). Figure 4 illustrates the relationship between $\mathrm{PCr}$ resynthesis rate and ADP in a representative subject and the fitted function from the mean parameters pre- and post-training.

\section{Metabolic changes and reproducibility of the results}

Table 2 summarizes intracellular metabolite concentrations and $\mathrm{pH}$ at the end of exercise and after a constant-load protocol in TC. In contrast to the training group, there was no systematic variation in the metabolic response to exercise and recovery between the two measurements performed two weeks apart demonstrating good reproducibility of the measurements employed in this study. 


\section{Discussion}

In this study, we used ${ }^{31} \mathrm{P}$-MRS to examine the effects of short-term high-intensity exercise training on the control of respiration rate by ADP and oxidative capacity in young healthy men during plantar flexion exercise. Short-term training attenuated the exercise-induced reduction in $\Delta \mathrm{G}_{\mathrm{ATP}}$ as well as $\mathrm{ADP}$ and $\mathrm{Pi}$ accumulation. In agreement with our hypothesis, 5 days of training was not associated with a consistent improvement in muscle oxidative capacity, but rather an increased sensitivity of mitochondrial respiration to ADP. Thus, documenting a rapid exercise-training induced adaptation in the allosteric regulation of mitochondrial respiration by ADP.

\section{Effects of short-term training on phosphorylated compounds during exercise}

In this study, we sought to examine the changes in phosphorylated compounds during exercise following high-intensity short-term training. Although PCr kinetics were unchanged, the fall in $\Delta \mathrm{G}_{\mathrm{ATP}}$ as well as ADP and Pi accumulation were substantially reduced, which illustrates an improved energetic state. In agreement with this conclusion, it has previously been reported that ADP and inosine monophosphate concentrations measured by biopsy were lower during steady-state exercise after a short-term endurance training period (Green et al., 1999; Green et al., 2000; Green et al., 2009). PCr concentration was also higher in these studies, which somewhat contrasts with our similar $\mathrm{PCr}$ concentrations pre- and post-training. However, some of these discrepancies could be a consequence of the differing durations of the exercise protocols (15 to $60 \mathrm{~min}$ versus $4 \mathrm{~min}$ in the current study). Together, these findings are consistent with a tighter metabolic control and a greater mitochondrial respiration sensitivity to metabolic feedback during exercise which is similar to what has been previously documented as a result of long-term endurance-training programs (Zoladz et al., 2006; Jones et al., 2007). Therefore, the present study extends this prior work revealing that the adaptation in muscle metabolism aimed at improving the energetic state during exercise occurs early (within 5 days of training) and without a measurable and consistent alteration in oxidative capacity.

\section{Effects of short-term training on muscle oxidative capacity}

As anticipated, 5 days of high-intensity training did not significantly affect muscle oxidative capacity, evidenced by the unaltered PCr recovery time constant and maximal rate of oxidative ATP synthesis (figure 3). Unlike the PCr recovery time constant which relies on end-exercise $\mathrm{pH}$, increasing its variability, Vmax is independent of the end-exercise condition (Roussel et al., 2000), making it a robust index of muscle oxidative capacity. Such conclusions are supported by the inclusion of a time control in the current study that demonstrated good reliability of these measurements (Table 2).

An important aspect of this study was that, despite different assumptions related to the metabolic control of respiration rate, the results and therefore the conclusions from the $\mathrm{PCr}$ recovery time constant and $V_{\max }$ analyses were similar: short-term training did not significantly improve muscle oxidative capacity. In addition, the values reported for the $\mathrm{PCr}$ recovery time constant $(\sim 30 \mathrm{~s})$ and $\mathrm{V}_{\max }\left(\sim 30 \mathrm{mM}\right.$. $\left.\mathrm{min}^{-1}\right)$ before and after the short-term training period are similar to those previously reported in calf muscle of young untrained subjects (Johansen \& Quistorff, 2003; Haseler et al., 2004). While, the PCr recovery time constant and $\mathrm{V}_{\max }$ were higher and lower, respectively, than that observed in endurancetrained subjects ( 25 s and $\sim 44 \mathrm{mM} . \mathrm{min}^{-1}$ ) (Haseler et al., 1999; Johansen \& Quistorff, 2003), which, in combination, further supports our conclusion that muscle oxidative capacity was not substantially improved. 


\section{Effects of short-term training on the control of mitochondrial respiration}

A major finding of this study was the documentation of improved cooperativity between ADP and mitochondrial respiration (figure 4), as exemplified by the enhanced Hill coefficient (from $\sim 2.7$ to $\sim 3.4$ post-training), before any noticeable improvement in the maximal rate of oxidative ATP synthesis. Such a finding has important implications in terms of both the control of mitochondrial respiration and metabolic adaptations to exercise training.

Among several potential mechanisms (Chance \& Williams, 1955; Meyer, 1988; Korzeniewski, 2004), it has been postulated that the control of respiration rate is mediated by ADP according to a higher order or allosteric activation model (Jeneson et al., 1996). Indeed, the sigmoid relationship between [ADP] and mitochondrial respiration rate documented in the present study (figure 4) is consistent with this allosteric activation model in vivo. This finding contrasts somewhat with the classic Michaelis-Menten model describing a hyperbolic relationship between respiration rate and [ADP] in isolated mitochondria (Chance \& Williams, 1955) and in permeabilized muscle fibers (Zoll et al., 2002; Gueguen et al., 2005). However, there is now accumulating evidence suggesting that simple first-order control models are not sufficient to predict the dynamics of respiration rate in vivo. For instance, in human skeletal muscle the Hill coefficient describing the relationship between respiration rate and [ADP] amounted $~ 2.0$ in human quadriceps and forearm, consistent with a second-order model (allosteric activation) (Jeneson et al., 1996; Jeneson et al., 2009). In addition, computer simulations of the regulation of mitochondrial oxidative phosphorylation provided values similar to the present study with the Hill coefficient ranging between 2.6 and 3.4 in skeletal muscle (Vicini \& Kushmerick, 2000). Recently, Wust et al. (Wust et al., 2011) even reported a Hill coefficient of 3.8 in highly oxidative canine gastrocnemius muscle in situ. Therefore, overall, including the current data, these findings suggest that respiratory control by allosteric activation provides a better characterisation than first-order control models of the dynamics of muscle respiration rate in vivo.

Consistent with our hypothesis, the sensitivity of mitochondrial respiration to ADP was significantly increased after training (figure 4) through an improvement in cooperativity (Hill coefficient). Interestingly, it has previously been suggested that variations in $\mathrm{O}_{2}$ availability can modulate the concentration of other reactants involved in the control of respiration rate (e.g. $\mathrm{PCr}, \mathrm{ADP}, \mathrm{Pi}, \mathrm{NADH})$, thus, altering mitochondrial sensitivity even when intracellular $\mathrm{PO}_{2}$ is above the level considered critical for mitochondrial respiration (Connett et al., 1990; Wilson, 1994). In support of this concept, using a canine hindlimb preparation, it has been demonstrated that varying the degree of tissue oxygenation at a given respiration rate elicited different concentrations in the proposed regulators of mitochondrial respiration (PCr, ADP, Pi) (Hogan et al., 1992a; Hogan et al., 1992b). In light of these studies, the observed improvement in the sensitivity of mitochondrial respiration to ADP in the current study could potentially be mediated by an increase in intracellular $\mathrm{PO}_{2}$ after training. Indeed, Green et al. (2012) recently demonstrated that short-term training induced a rapid increase in the capillary to fiber area ratio after only 3 days of training (Green et al., 2012) suggestive of an enhanced $\mathrm{O}_{2}$ diffusing capacity and subsequently increased $\mathrm{O}_{2}$ availability in the tissue. Together, these findings could account for the attenuated ADP and Pi accumulation observed at the end of exercise (table 1) and are consistent with a tighter metabolic control of oxidative ATP synthesis.

Traditionally it is thought that the increase in mitochondrial respiration capacity associated with exercise training leads to smaller relative changes in the cytosolic modulators of oxidative phosphorylation (NADH, ADP, Pi and PCr), implying increased sensitivity of 
mitochondrial respiration (Dudley et al., 1987). In this respect, the results of the present study provide novel insights into the mechanism responsible for skeletal muscle adaptation to exercise training by revealing that in the early stages of such training the sensitivity of mitochondrial respiration is increased before a change in mitochondrial respiration capacity (or mitochondrial density). In fact, this finding is supported by recent results in permeabilized fibers, demonstrating that a greater respiration sensitivity can occur even after just a single exercise session, independently of any change in mitochondrial content or fiber type (Perry et al., 2012).

Although highly speculative at this point, the mechanisms by which mitochondrial respiration-[ADP] cooperativity was improved by short-term training may stem from rapid modifications in the structural arrangement of mitochondria around myofibrils or improved functional coupling between mitochondrial ATP production and sites of ATP consumption through the creatine kinase system. One mechanism potentially explaining an increase in the Hill coefficient post-training may relate to the compartmentalization of energy exchange, which restricts the access of exogenous ADP to mitochondria. Indeed, it has been suggested that mitochondria, sarcoplasmic reticulum and myofibrils may be associated functionally in skeletal muscle (Seppet et al., 2001), such that the ADP produced during intracellular ATPase reactions (myosin- and calcium-ATPases) would be directly channeled to the mitochondria. In support of this, similar mitochondrial respiration rates in permeabilized fibers can be achieved with 40 times less ADP derived from myosin- and calcium-ATPases activity compared to directly adding ADP to the incubation medium (Kaasik et al., 2001). Therefore, one can speculate that structural arrangement of the mitochondria around myofibrils might occur rapidly after just a few days of exercise training improving the energy channeling within these functional units. An alternative mechanism explaining our result may involve the $\mathrm{Cr}-\mathrm{PCr}$ shuttle, which has been suggested to play a key role in the regulation of mitochondrial oxidation (Mahler, 1985). In fact, up to $80 \%$ of the energy transfer from the matrix of the mitochondria to the cytoplasm has been proposed to occur through this process rather than diffusion of adenine nucleotide (Aliev et al.). According to this model, ATP produced within the matrix is transported to the inter-membrane space by adenine nucleotide translocase (ANT) where phosphate transfers to $\mathrm{Cr}$ through mitochondrial creatine kinase (mi-CK). The newly formed $\mathrm{PCr}$ is then transported through voltage dependent anion channel (VDAC), located on the outer mitochondrial membrane, to the cytosol. After that, $\mathrm{PCr}$ is used by cytosolic creatine kinase reaction to regenerate ATP locally at the ATP consuming sites (myosin- and calcium-ATPases) (Aliev et al.). Interestingly, mi-CK sensitivity to Cr appears to be increased after an acute bout of exercise (Perry et al., 2012) suggesting that the improvement in mitochondrial respiration- [ADP] cooperativity observed herein may be secondary to an increase in mi-CK sensitivity. Additional studies using in vitro approaches are therefore warranted to further investigate these mechanisms potentially altering mitochondrial respiration control with training.

\section{Methodological considerations}

One could suggest that our exercise training may have resulted in a loss of [ATP], therefore affecting our assumption about a constant [ATP] throughout the protocol. However, in studies that reported such a phenomenon subjects underwent exercise training at a much higher intensity (sprint training) yielding greater metabolic perturbation in comparison to our high-intensity aerobic exercise (Hellsten-Westing et al., 1993; Stathis et al., 1994; Hellsten et al., 1998; Burgomaster et al., 2006). In these prior studies, the training programs were actually designed to result in a loss of adenine nucleotides for several days by inducing subject exhaustion and formation of inosine monophosphate (IMP) and ammonia, and reducing adenine nucleotide repletion (Hellsten et al., 1998). In stark contrast, our exercise intensity was set in the aerobic heavy intensity domain (85\% of WRmax measured during an 
incremental aerobic protocol) (Whipp et al., 2005). Using a similar type of aerobic training, several studies have documented that [ATP] is maintained for several days after such a regimen (2 hrs at 67\% of VO2max, 5-7 days consecutive days) (Green et al., 1992; Cadefau et al., 1994; Phillips et al., 1995). Therefore, it is very unlikely that [ATP] was altered in the current study.

In the present study, it was assumed that PCr represents $85 \%$ of the total creatine content, which may have some bearing on the analysis of the control of respiration. First, in terms of absolute quantification, the resting concentration of PCr reported here $(32 \pm 3 \mathrm{mM})$ are in line with previous studies that have either utilized assumptions about $\mathrm{TCr}(\sim 33 \mathrm{mM})$ or calibrated ${ }^{31} \mathrm{P}-\mathrm{MRS}$, i.e. with an external reference or ${ }^{1} \mathrm{H}-\mathrm{MRS},(34 \pm 1 \mathrm{mM})($ Kemp et al., 2007). Although lower values for resting $[\mathrm{PCr}]$ has been previously reported using biochemical methods from biopsies of the calf (26-30 mM/L) in comparison to ${ }^{31} \mathrm{P}-\mathrm{MRS}$ (Bangsbo et al., 1993), these authors concluded at a reasonable agreement between these methods. In fact, it has been argued that most of the differences between these methods can be attributed to the delay between sampling and freezing during which time $\mathrm{PCr}$ continues to be broken down by creatine kinase resulting in lower [PCr] with biochemical methods (Kemp et al., 2007). In support of this explanation, Bangsbo et al. (Bangsbo et al., 1993) reported that the lower $\mathrm{PCr}$ in the biochemical analysis was completely accounted for by a higher Pi concentration. This bias in the biochemical method would also explain the difference with the $\mathrm{PCr} / \mathrm{TCr}$ ratio $(\sim 61 \%)$ calculated from a previous study (Harris et al., 1974). In fact, based upon a PCr/TCr ratio of $61 \%(41.5 \mathrm{mM} / \mathrm{L}$ for TCr and $25 \mathrm{mM} / \mathrm{L}$ for $\mathrm{PCr}$ ) (Harris et al., 1974) and a resting $\mathrm{pH}$ of 7.03 (data from the present study), resting [ADP] would be $\sim 34 \mu \mathrm{M}$, which is incompatible with previous values reported in the calf using either biochemical ( 8 $\mu \mathrm{M}$ ) (Bangsbo et al., 1993) or ${ }^{31}$ P-MRS methodologies ( 8-12 $\mu \mathrm{M})$ [(Lodi et al., 1998) and present study]. Finally, based upon computer simulation, Kemp et al. (Kemp et al., 2007) reported that a decrease in [PCr] would further increase the Hill coefficient, supporting our conclusion that respiratory control by allosteric activation provides a better characterisation than first-order control models of the dynamics of muscle respiration rate in vivo.

Conceptually, exercise training per se can change motor unit recruitment patterns (Ploutz et al., 1994) and therefore potentially alter metabolic capacity (McCully et al., 2011). However, Walter et al. (Walter et al., 1997) previously reported that, in humans, an alteration in the number of motor units recruited, achieved by two protocols known to elicit different fiber type recruitment patterns during voluntary contractions, does not alter muscle oxidative capacity as measured by ${ }^{31} \mathrm{P}$-MRS. Therefore, it is also unlikely that any potential change in motor unit recruitment, induced by the current short-term endurance training regimen, would affect our conclusions.

With regards to the confidence in the kinetics parameters estimated by the fitting, the confidence intervals were relatively narrow as illustrated by the low CV $(\sim 10-20 \%)$. Specifically, the Hill coefficient exhibited a coefficient of variation of $17 \pm 14 \%$ and $13 \pm$ $14 \%$ pre- and post-training respectively, in conjunction with an IC95 of $0.9 \pm 0.6$ and $0.9 \pm$ 0.3 pre- and post-training respectively. Together, these indices indicate reasonable confidence in the fitting parameters with the current protocol. These values are quite remarkable considering that our subjects performed only a single exercise test. In contrast, similar values could only be obtained for $\mathrm{VO}_{2}$ kinetics, assessed at the mouth, by combining several repetitions (Lamarra et al., 1987) or re-sampling the original dataset (Borrani et al., 2001). Additionally, the fact that, $n \mathrm{H}$ was increased by $28 \%$ (i.e. well above the variability associated with the modeling of the [ADP]-respiration rate relationship) strongly supports our conclusion that this parameter was improved by the short-term training. 


\section{Conclusion}

In summary, 5 days of exercise training resulted in an improvement in exercise capacity and an improved cooperativity between mitochondrial respiration and ADP before any significant alteration in the maximal rate of oxidative ATP synthesis. These alterations were also associated with an attenuated fall in $\Delta \mathrm{G}_{\mathrm{ATP}}$ as well as a reduced accumulation of ADP and Pi during exercise at the same absolute workload. Thus, this study provides evidence that a short-term training program can induce a rapid adaptation in the allosteric control of respiration rate by ADP, potentially mediated by an increase in $\mathrm{O}_{2}$ availability, before any measurable changes in muscle oxidative capacity.

\section{Acknowledgments}

The Authors wish to thank all the subjects in this study for their committed participation in this research. This work was funded in part by grants from the NIH National Heart, Lung, and Blood Institute (PO1 HL 09183), TobaccoRelated Disease Research Program Grant (15RT-0100) and VA Merit grant E6910R.

\section{Bibliography}

Aliev M, Guzun R, Karu-Varikmaa M, Kaambre T, Wallimann T, Saks V. Molecular system bioenergics of the heart: experimental studies of metabolic compartmentation and energy fluxes versus computer modeling. International journal of molecular sciences. 12:9296-9331. [PubMed: 22272134]

Bangsbo J, Johansen L, Quistorff B, Saltin B. NMR and analytic biochemical evaluation of CrP and nucleotides in the human calf during muscle contraction. J Appl Physiol. 1993; 74:2034-2039. [PubMed: 8514726]

Borrani F, Candau R, Millet GY, Perrey S, Fuchslocher J, Rouillon JD. Is the VO2 slow component dependent on progressive recruitment of fast-twitch fibers in trained runners? J Appl Physiol. 2001; 90:2212-2220. [PubMed: 11356785]

Burgomaster KA, Heigenhauser GJ, Gibala MJ. Effect of short-term sprint interval training on human skeletal muscle carbohydrate metabolism during exercise and time-trial performance. J Appl Physiol. 2006; 100:2041-2047. [PubMed: 16469933]

Cadefau J, Green HJ, Cusso R, Ball-Burnett M, Jamieson G. Coupling of muscle phosphorylation potential to glycolysis during work after short-term training. J Appl Physiol. 1994; 76:2586-2593. [PubMed: 7928887]

Chance B, Williams GR. Respiratory enzymes in oxidative phosphorylation. I. Kinetics of oxygen utilization. The Journal of biological chemistry. 1955; 217:383-393. [PubMed: 13271402]

Conley KE, Kushmerick MJ, Jubrias SA. Glycolysis is independent of oxygenation state in stimulated human skeletal muscle in vivo. The Journal of physiology. 1998; 511(Pt 3):935-945. [PubMed: 9714871]

Connett RJ, Honig CR, Gayeski TE, Brooks GA. Defining hypoxia: a systems view of VO2, glycolysis, energetics, and intracellular PO2. J Appl Physiol. 1990; 68:833-842. [PubMed: 2187852]

Delp MD. Differential effects of training on the control of skeletal muscle perfusion. Medicine and science in sports and exercise. 1998; 30:361-374. [PubMed: 9526881]

Dudley GA, Tullson PC, Terjung RL. Influence of mitochondrial content on the sensitivity of respiratory control. The Journal of biological chemistry. 1987; 262:9109-9114. [PubMed: 3597408]

Golding EM, Teague WE Jr, Dobson GP. Adjustment of K' to varying $\mathrm{pH}$ and $\mathrm{pMg}$ for the creatine kinase, adenylate kinase and ATP hydrolysis equilibria permitting quantitative bioenergetic assessment. The Journal of experimental biology. 1995; 198:1775-1782. [PubMed: 7636446]

Green H, Grant S, Bombardier E, Ranney D. Initial aerobic power does not alter muscle metabolic adaptations to short-term training. The American journal of physiology. 1999; 277:E39-48. [PubMed: 10409126] 
Green H, Tupling R, Roy B, O’Toole D, Burnett M, Grant S. Adaptations in skeletal muscle exercise metabolism to a sustained session of heavy intermittent exercise. Am J Physiol Endocrinol Metab. 2000; 278:E118-126. [PubMed: 10644545]

Green HJ, Bombardier E, Burnett ME, Smith IC, Tupling SM, Ranney DA. Time-dependent effects of short-term training on muscle metabolism during the early phase of exercise. American journal of physiology. 2009; 297:R1383-1391. [PubMed: 19710384]

Green HJ, Burnett M, Kollias H, Ouyang J, Smith I, Tupling S. Can increases in capillarization explain the early adaptations in metabolic regulation in human muscle to short-term training? Canadian journal of physiology and pharmacology. 2012; 90:557-566. [PubMed: 22471991]

Green HJ, Helyar R, Ball-Burnett M, Kowalchuk N, Symon S, Farrance B. Metabolic adaptations to training precede changes in muscle mitochondrial capacity. J Appl Physiol. 1992; 72:484-491. [PubMed: 1559923]

Green HJ, Jones S, Ball-Burnett ME, Smith D, Livesey J, Farrance BW. Early muscular and metabolic adaptations to prolonged exercise training in humans. J Appl Physiol. 1991; 70:2032-2038. [PubMed: 1864784]

Gueguen N, Lefaucheur L, Fillaut M, Herpin P. Muscle fiber contractile type influences the regulation of mitochondrial function. Molecular and cellular biochemistry. 2005; 276:15-20. [PubMed: 16132680]

Harris RC, Hultman E, Nordesjo LO. Glycogen, glycolytic intermediates and high-energy phosphates determined in biopsy samples of musculus quadriceps femoris of man at rest. Methods and variance of values. Scandinavian journal of clinical and laboratory investigation. 1974; 33:109_ 120. [PubMed: 4852173]

Haseler LJ, Hogan MC, Richardson RS. Skeletal muscle phosphocreatine recovery in exercise-trained humans is dependent on O2 availability. J Appl Physiol. 1999; 86:2013-2018. [PubMed: 10368368]

Haseler LJ, Lin AP, Richardson RS. Skeletal muscle oxidative metabolism in sedentary humans: 31PMRS assessment of O2 supply and demand limitations. J Appl Physiol. 2004; 97:1077-1081. [PubMed: 15133010]

Helgerud J, Hoydal K, Wang E, Karlsen T, Berg P, Bjerkaas M, Simonsen T, Helgesen C, Hjorth N, Bach R, Hoff J. Aerobic high-intensity intervals improve VO2max more than moderate training. Medicine and science in sports and exercise. 2007; 39:665-671. [PubMed: 17414804]

Hellsten-Westing Y, Norman B, Balsom PD, Sjodin B. Decreased resting levels of adenine nucleotides in human skeletal muscle after high-intensity training. J Appl Physiol. 1993; 74:2523-2528. [PubMed: 8335586]

Hellsten Y, Sjodin B, Richter EA, Bangsbo J. Urate uptake and lowered ATP levels in human muscle after high-intensity intermittent exercise. The American journal of physiology. 1998; 274:E600606. [PubMed: 9575819]

Hogan MC, Arthur PG, Bebout DE, Hochachka PW, Wagner PD. Role of O2 in regulating tissue respiration in dog muscle working in situ. J Appl Physiol. 1992a; 73:728-736. [PubMed: 1400003]

Hogan MC, Nioka S, Brechue WF, Chance B. A 31P-NMR study of tissue respiration in working dog muscle during reduced O2 delivery conditions. J Appl Physiol. 1992b; 73:1662-1670. [PubMed: 1447118]

Holloszy JO, Coyle EF. Adaptations of skeletal muscle to endurance exercise and their metabolic consequences. J Appl Physiol. 1984; 56:831-838. [PubMed: 6373687]

Jeneson JA, Schmitz JP, van den Broek NM, van Riel NA, Hilbers PA, Nicolay K, Prompers JJ. Magnitude and control of mitochondrial sensitivity to ADP. Am J Physiol Endocrinol Metab. 2009; 297:E774-784. [PubMed: 19622784]

Jeneson JA, Westerhoff HV, Brown TR, Van Echteld CJ, Berger R. Quasi-linear relationship between Gibbs free energy of ATP hydrolysis and power output in human forearm muscle. The American journal of physiology. 1995; 268:C1474-1484. [PubMed: 7611368]

Jeneson JA, Wiseman RW, Westerhoff HV, Kushmerick MJ. The signal transduction function for oxidative phosphorylation is at least second order in ADP. The Journal of biological chemistry. 1996; 271:27995-27998. [PubMed: 8910406] 
Johansen L, Quistorff B. 31P-MRS characterization of sprint and endurance trained athletes. International journal of sports medicine. 2003; 24:183-189. [PubMed: 12740736]

Jones AM, Carter $\mathrm{H}$. The effect of endurance training on parameters of aerobic fitness. Sports medicine (Auckland, NZ. 2000; 29:373-386.

Jones AM, Wilkerson DP, Berger NJ, Fulford J. Influence of endurance training on muscle [PCr] kinetics during high-intensity exercise. Am J Physiol Regul Integr Comp Physiol. 2007; 293:R392-401. [PubMed: 17475681]

Kaasik A, Veksler V, Boehm E, Novotova M, Minajeva A, Ventura-Clapier R. Energetic crosstalk between organelles: architectural integration of energy production and utilization. Circulation research. 2001; 89:153-159. [PubMed: 11463722]

Kemp GJ, Meyerspeer M, Moser E. Absolute quantification of phosphorus metabolite concentrations in human muscle in vivo by 31P MRS: a quantitative review. NMR in biomedicine. 2007; 20:555565. [PubMed: 17628042]

Kemp GJ, Roussel M, Bendahan D, Le Fur Y, Cozzone PJ. Interrelations of ATP synthesis and proton handling in ischaemically exercising human forearm muscle studied by 31P magnetic resonance spectroscopy. The Journal of physiology. 2001; 535:901-928. [PubMed: 11559784]

Korzeniewski B. The modeling of oxidative phosphorylation in skeletal muscle. Jpn J Physiol. 2004; 54:511-516. [PubMed: 15760482]

Lamarra N, Whipp BJ, Ward SA, Wasserman K. Effect of interbreath fluctuations on characterizing exercise gas exchange kinetics. J Appl Physiol. 1987; 62:2003-2012. [PubMed: 3110126]

Lodi R, Taylor DJ, Tabrizi SJ, Hilton-Jones D, Squier MV, Seller A, Styles P, Schapira AH. Normal in vivo skeletal muscle oxidative metabolism in sporadic inclusion body myositis assessed by $31 \mathrm{P}$ magnetic resonance spectroscopy. Brain. 1998; 121(Pt 11):2119-2126. [PubMed: 9827771]

Mahler M. First-order kinetics of muscle oxygen consumption, and an equivalent proportionality between QO2 and phosphorylcreatine level. Implications for the control of respiration. J Gen Physiol. 1985; 86:135-165. [PubMed: 4031824]

McCully KK, Mulcahy TK, Ryan TE, Zhao Q. Skeletal muscle metabolism in individuals with spinal cord injury. J Appl Physiol. 2011; 111:143-148. [PubMed: 21512153]

Meyer RA. A linear model of muscle respiration explains monoexponential phosphocreatine changes. The American journal of physiology. 1988; 254:C548-553. [PubMed: 3354652]

Motulsky, HJ.; Christopoulos, A. Fitting models to biological data using linear and nonlinear regression. A practical guide to curve fitting. San Diego: 2003.

Perry CG, Kane DA, Herbst EA, Mukai K, Lark DS, Wright DC, Heigenhauser GJ, Neufer PD, Spriet LL, Holloway GP. Mitochondrial Creatine Kinase Activity and Phosphate Shuttling Are Acutely Regulated by Exercise in Human Skeletal Muscle. The Journal of physiology. 2012

Phillips SM, Green HJ, Tarnopolsky MA, Grant SM. Increased clearance of lactate after short-term training in men. J Appl Physiol. 1995; 79:1862-1869. [PubMed: 8847245]

Ploutz LL, Tesch PA, Biro RL, Dudley GA. Effect of resistance training on muscle use during exercise. J Appl Physiol. 1994; 76:1675-1681. [PubMed: 8045847]

Putman CT, Jones NL, Hultman E, Hollidge-Horvat MG, Bonen A, McConachie DR, Heigenhauser GJ. Effects of short-term submaximal training in humans on muscle metabolism in exercise. The American journal of physiology. 1998; 275:E132-139. [PubMed: 9688884]

Roussel M, Bendahan D, Mattei JP, Le Fur Y, Cozzone PJ. 31P magnetic resonance spectroscopy study of phosphocreatine recovery kinetics in skeletal muscle: the issue of intersubject variability. Biochimica et biophysica acta. 2000; 1457:18-26. [PubMed: 10692546]

Seppet EK, Kaambre T, Sikk P, Tiivel T, Vija H, Tonkonogi M, Sahlin K, Kay L, Appaix F, Braun U, Eimre M, Saks VA. Functional complexes of mitochondria with Ca, MgATPases of myofibrils and sarcoplasmic reticulum in muscle cells. Biochimica et biophysica acta. 2001; 1504:379-395. [PubMed: 11245802]

Spina RJ, Chi MM, Hopkins MG, Nemeth PM, Lowry OH, Holloszy JO. Mitochondrial enzymes increase in muscle in response to 7-10 days of cycle exercise. J Appl Physiol. 1996; 80:2250 2254. [PubMed: 8806937]

Starritt EC, Angus D, Hargreaves M. Effect of short-term training on mitochondrial ATP production rate in human skeletal muscle. J Appl Physiol. 1999; 86:450-454. [PubMed: 9931175] 
Stathis CG, Febbraio MA, Carey MF, Snow RJ. Influence of sprint training on human skeletal muscle purine nucleotide metabolism. J Appl Physiol. 1994; 76:1802-1809. [PubMed: 8045862]

Taylor DJ, Bore PJ, Styles P, Gadian DG, Radda GK. Bioenergetics of intact human muscle. A 31P nuclear magnetic resonance study. Molecular biology \& medicine. 1983; 1:77-94. [PubMed: 6679873]

Thompson CH, Kemp GJ, Sanderson AL, Radda GK. Skeletal muscle mitochondrial function studied by kinetic analysis of postexercise phosphocreatine resynthesis. J Appl Physiol. 1995; 78:21312139. [PubMed: 7665409]

Vicini P, Kushmerick MJ. Cellular energetics analysis by a mathematical model of energy balance: estimation of parameters in human skeletal muscle. American journal of physiology. 2000; 279:C213-224. [PubMed: 10898733]

Walter G, Vandenborne K, McCully KK, Leigh JS. Noninvasive measurement of phosphocreatine recovery kinetics in single human muscles. Am J Physiol. 1997; 272:C525-534. [PubMed: 9124295]

Whipp BJ, Ward SA, Rossiter HB. Pulmonary O2 uptake during exercise: conflating muscular and cardiovascular responses. Medicine and science in sports and exercise. 2005; 37:1574-1585. [PubMed: 16177611]

Wilson DF. Factors affecting the rate and energetics of mitochondrial oxidative phosphorylation. Medicine and science in sports and exercise. 1994; 26:37-43. [PubMed: 8133736]

Wust RC, Grassi B, Hogan MC, Howlett RA, Gladden LB, Rossiter HB. Kinetic control of oxygen consumption during contractions in self-perfused skeletal muscle. The Journal of physiology. 2011

Zoladz JA, Korzeniewski B, Grassi B. Training-induced acceleration of oxygen uptake kinetics in skeletal muscle: the underlying mechanisms. J Physiol Pharmacol. 2006; 57(Suppl 10):67-84. [PubMed: 17242492]

Zoll J, Sanchez H, N'Guessan B, Ribera F, Lampert E, Bigard X, Serrurier B, Fortin D, Geny B, Veksler V, Ventura-Clapier R, Mettauer B. Physical activity changes the regulation of mitochondrial respiration in human skeletal muscle. The Journal of physiology. 2002; 543:191200. [PubMed: 12181291] 


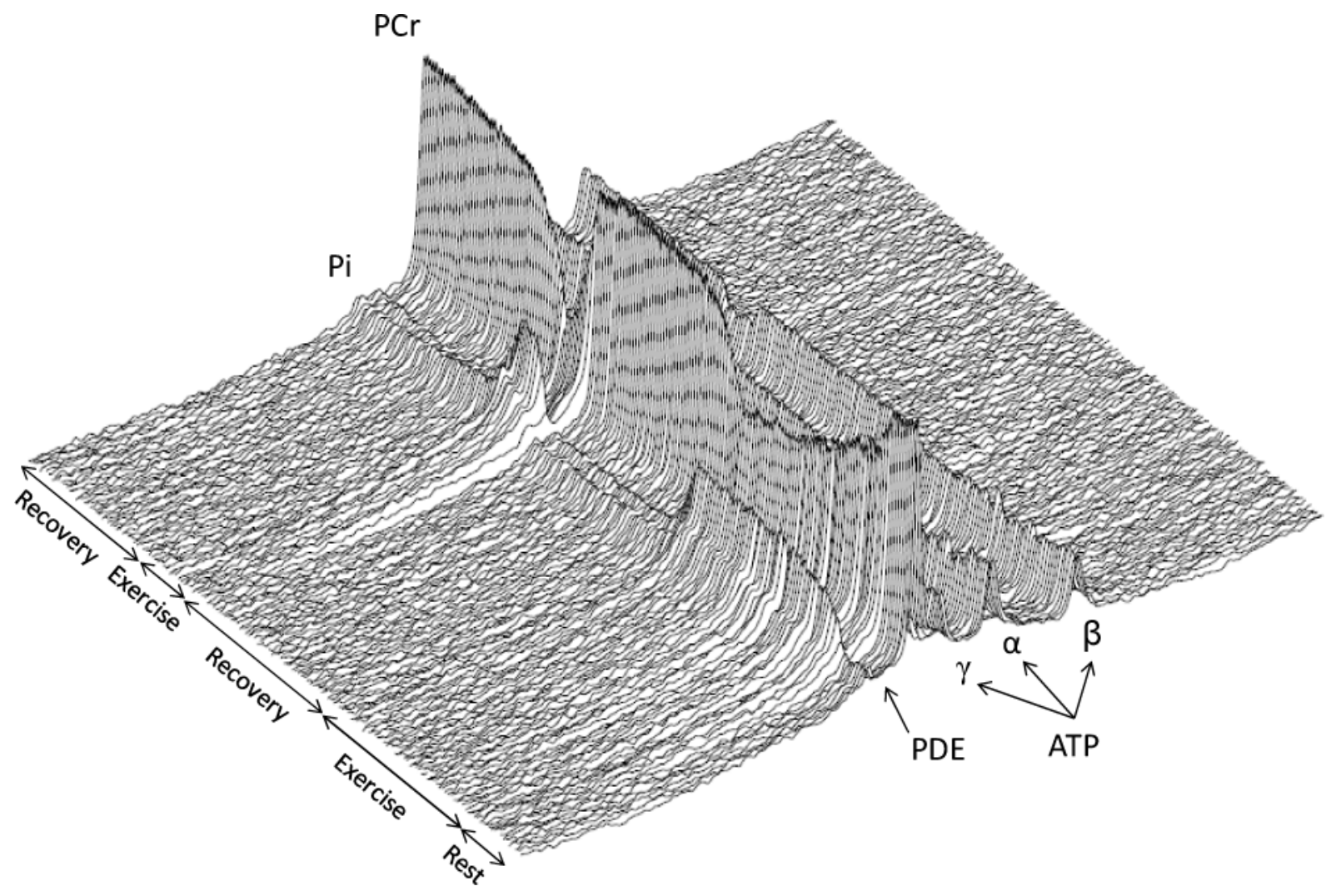

Figure 1.

Typical stack plot of ${ }^{31} \mathrm{P}$ spectra acquired from the plantar flexor muscles of one subject during a rest-exercise-recovery protocol. Pi: inorganic phosphate, PDE: Phosphodiester, PCr: Phosphocreatine, ATP: Adenosine Triphosphate, $\alpha, \beta, \gamma$ : phosphate groups of ATP. 


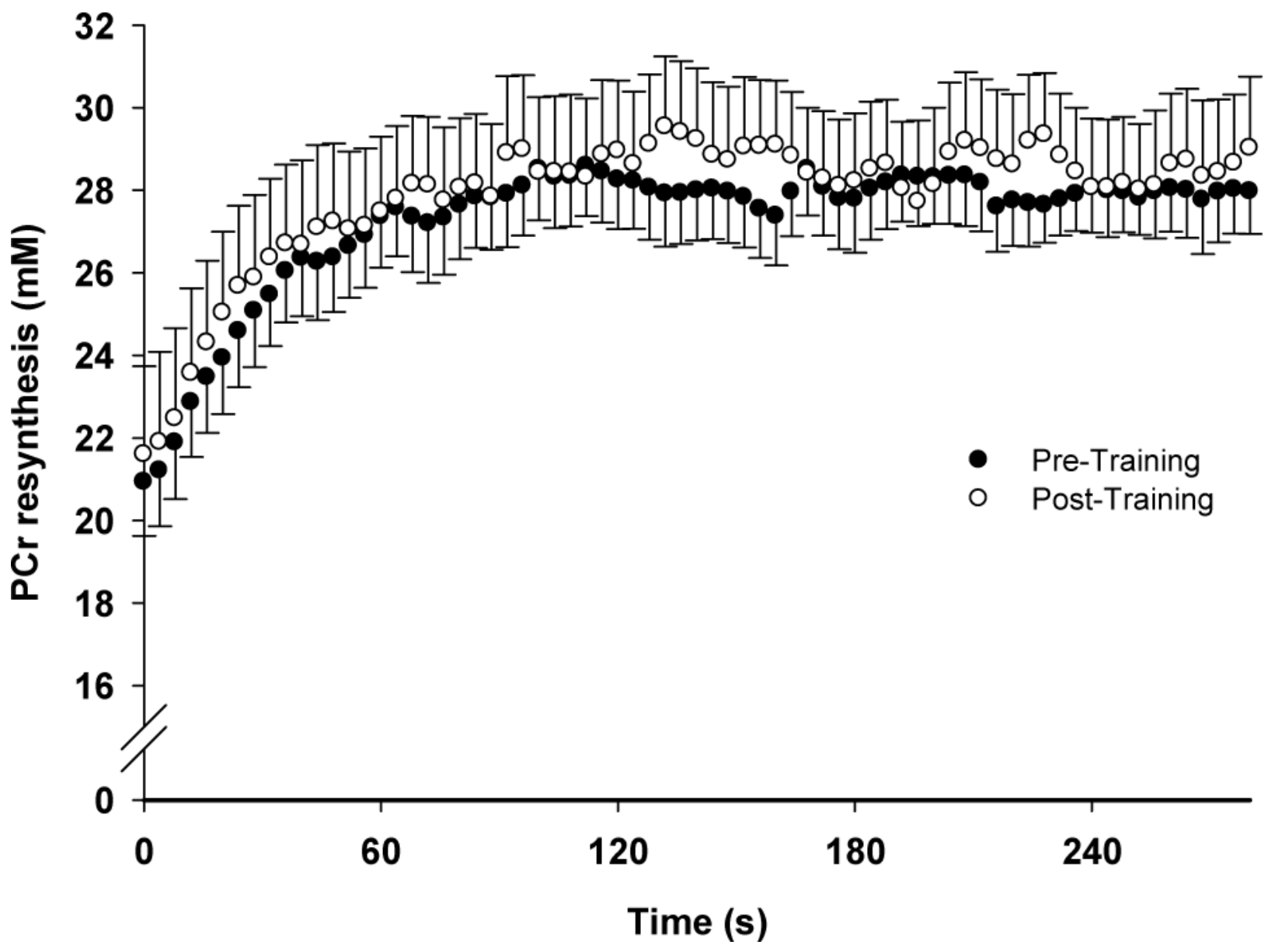

Figure 2.

Phosphocreatine dynamics during the recovery period pre- and post-training. Data are presented as mean \pm SEM. 

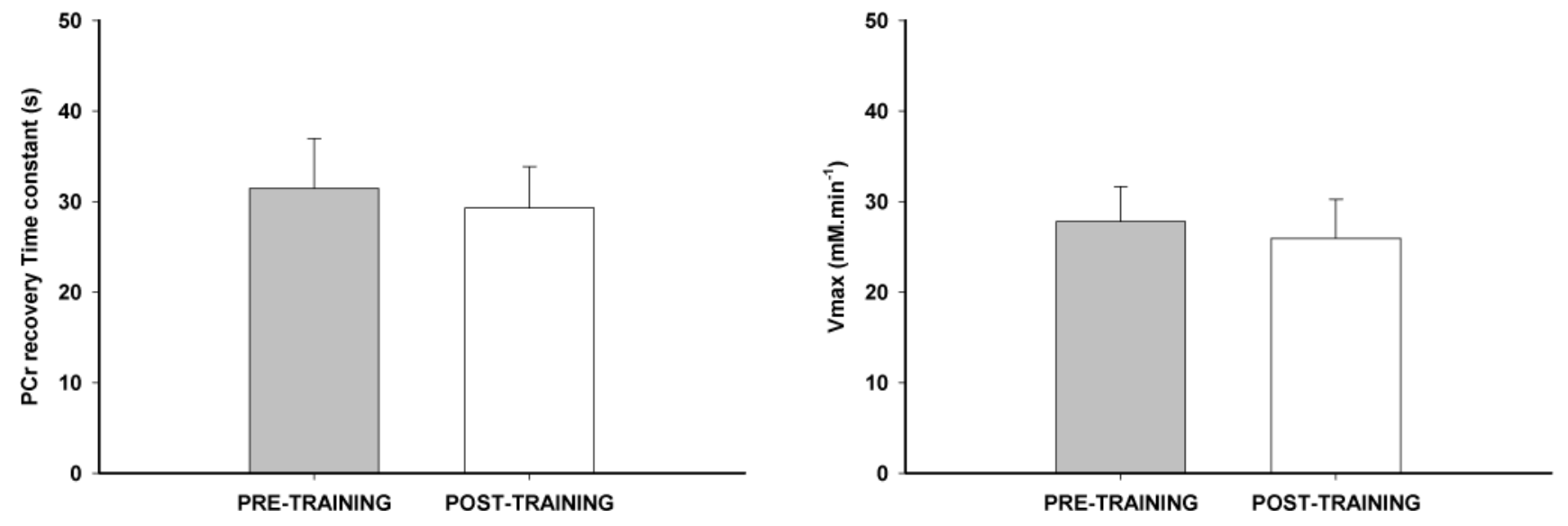

Figure 3.

The effect of 5 days of plantar flexion exercise training on muscle oxidative capacity assessed by phosphocreatine recovery time constant (left panel) and the maximal rate of oxidative ATP synthesis following exercise (right panel). Data are presented as mean \pm SEM. Both the PCr offset time constant and the maximal rate of oxidative ATP synthesis were not significantly different before and after 5 days of training $(P>0.05)$. 

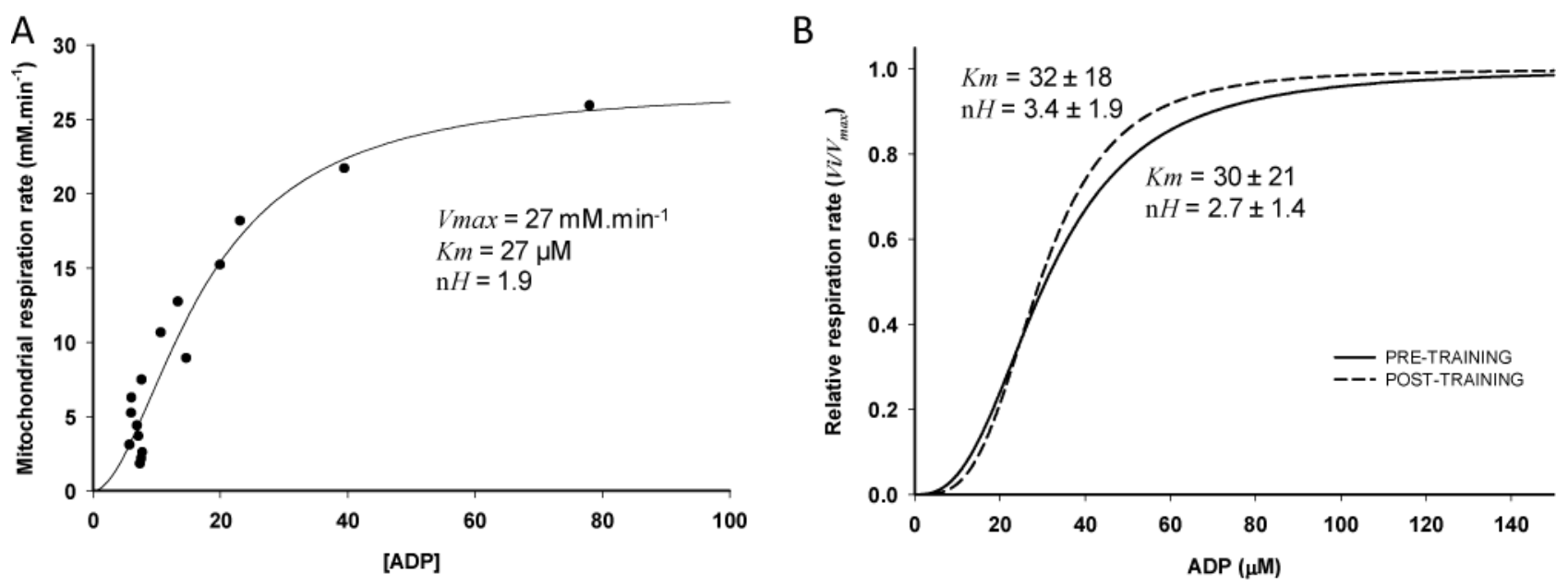

Figure 4.

Example of the pre-training $\mathrm{PCr}$ resynthesis rate with respect to ADP concentration fitted according to a sigmoid function with $\mathrm{K}_{\mathrm{m}}=27 \mu \mathrm{M}, n H=1.9$, the maximal rate of oxidative ATP synthesis $=27 \mathrm{mM} \cdot \mathrm{min}^{-1}$ and $\mathrm{r}^{2}=0.93(\mathrm{~A})$, and an illustration of the mean relative changes in respiration rate with respect to ADP concentration obtained from the fitted parameters pre- and post-plantar flexion training $(\mathrm{B}), n_{H}$ is the mean Hill coefficient pre and post-training. 


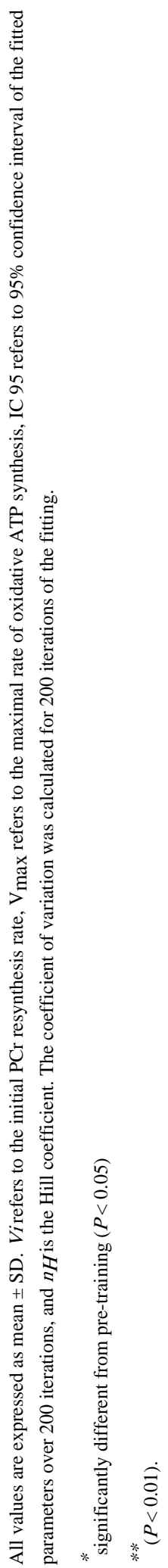


